\title{
Cytotoxic Chemotherapy as First-Line Therapy for Advanced Non-Small-Cell Lung Cancer in Taiwan: Daily Practice
}

\author{
Yi-Hsin Liang1,2,5, Yu-Yun Shao,4,5, Bin-Chi Liao 2,4, Ho-Sheng Lee9 , James Chih-Hsin Yang2,3,4,5, Ho-Min \\ Chen², Chun-Ju Chiang7,8, Ann-Lii Cheng2,4,5,6, Mei-Shu Lai ${ }^{7,8}$ \\ 1. Department of Oncology, National Taiwan University Hospital, Hsin-Chu Branch, Hsinchu, Taiwan \\ 2. Department of Oncology, National Taiwan University Hospital, Taipei, Taiwan \\ 3. Medical Research, National Taiwan University Hospital, Taipei, Taiwan \\ 4. National Taiwan University Cancer Center, Taipei, Taiwan \\ 5. Graduate Institute of Oncology, National Taiwan University College of Medicine \\ 6. Department of Internal Medicine, National Taiwan University College of Medicine \\ 7. Institute of Epidemiology and Preventive Medicine, College of Public Health, National Taiwan University, Taipei, Taiwan \\ 8. Taiwan Cancer Registry, Department of Internal Medicine, E-Da Hospital, Kaohsiung, Taiwan \\ 9. Division of Pulmonary Medicine, Department of Internal Medicine, E-Da Hospital, Kaohsiung, Taiwan \\ $\square$ Corresponding author: Mei-Shu Lai, MD, PhD, Institute of Epidemiology and Preventive Medicine, College of Public Health, National Taiwan University, \\ Room 518, No.17, Xuzhou Rd., Taipei City 10055, Taiwan; Phone: +886 2 33668018; Fax: +886 2 2351173; E-mail: mslai@cph.ntu.edu.tw
}

(1) Ivyspring International Publisher. Reproduction is permitted for personal, noncommercial use, provided that the article is in whole, unmodified, and properly cited. See http://ivyspring.com/terms for terms and conditions.

Received: 2016.02.03; Accepted: 2016.05.24; Published: 2016.07.07

\begin{abstract}
Aim: Cytotoxic chemotherapy is the standard first-line therapy for patients with advanced non-small cell lung cancer (NSCLC) without specific gene alterations. This study examined the prescription pattern and the survival outcome of cytotoxic chemotherapy regimens in daily practice in Taiwan.

Methods: We established a population-based cohort of patients diagnosed with advanced NSCLC between 2005 and 2009 using the databases of Taiwan Cancer Registry and National Health Insurance in Taiwan. We then analyzed chemotherapy prescriptions and the survival outcomes of patients.

Results: A total of 25,008 patients with advanced NSCLC were identified, 17,443 (70.0\%) of which received first-line chemotherapy and were therefore included in this study. Among them, 11,551 (66.2\%) patients had adenocarcinoma and 3,292 (18.9\%) patients had squamous cell carcinoma (SCC). Approximately $70 \%$ of the patients were diagnosed with NSCLC in medical centers. Platinum-based doublet chemotherapy was administered to $66.9 \%$ of the patients. Among all chemotherapy regimens, platinum with gemcitabine (33.8\%) was the most common, irrespective of geographic region. The second and third most common regimens were vinorelbine alone $(13.0 \%)$ and platinum with docetaxel (11.6\%). The prevalence of platinum-based doublet chemotherapy regimens decreased from $71.4 \%$ in 2005 to $64.1 \%$ in 2009 . Among patients with adenocarcinoma histology, those who received platinum with pemetrexed had longer OS than did patients who received other platinum-based regimens $(p<$ 0.001).

Conclusion: Our findings reaffirm that in real-world practice, treatment plans of advanced NSCLC should be drawn up according to histology type.
\end{abstract}

Key words: adenocarcinoma; chemotherapy; non-small cell lung cancer; first line; Squamous cell carcinoma

\section{Introduction}

Lung cancer is the leading cause of cancer-related death in Taiwan and around the world. Lung cancer is generally divided into two separate disease entities: small cell lung cancer and non-small

cell lung cancer (NSCLC)[1-3]. Chemotherapy is the primary treatment for advanced NSCLC. Considerable advances in targeted chemotherapy have been made in the treatment of patients with 
NSCLC harboring specific gene alterations, such as epidermal growth factor receptor (EGFR) mutations and anaplastic lymphoma kinase (ALK) translocations [4, 5]. The overall survival (OS) of patients with advanced NSCLC has significantly improved since 2000[6, 7].

However, for NSCLC patients without those specific gene alterations, cytotoxic chemotherapy remains the key therapy[8-16]. Platinum-based doublet chemotherapy is the current standard first-line chemotherapy for these patients[10, 16-18]. Gemcitabine, vinorelbine, docetaxel, paclitaxel, and pemetrexed are standard chemotherapeutic agents coupled with platinum. Previous clinical trials have demonstrated similar efficacy among these diverse first-line chemotherapy regimens. However, these studies were conducted before the emergence of targeted therapy and did not reflect actual clinical practice, especially in Taiwan.

We previously demonstrated that different first-line chemotherapy regimens resulted in similar survival outcomes among patients with advanced lung squamous cell carcinoma (SCC) using three national databases in Taiwan[19]. In this study, we took this investigation one step further by exploring all types of NSCLC histology with the aim of identifying the various treatment patterns employed in daily practice in Taiwan.

\section{Materials and Methods}

\section{Database}

We established a population-based cohort linking three national databases. This included the Taiwan Cancer Registry Database (TCRD), which is managed by the Health Promotion Administration, Ministry of Health and Welfare, Taiwan[20, 21]. All major cancer care providers in Taiwan are obligated to input patient data into the TCRD, including patient demographics, histology types, and the initial staging. This made it possible to retrieve all of the clinicopathologic characteristics of the patients from the TCRD. The second database was the claims database of the Taiwan National Health Insurance (NHI), which is a mandatory health insurance system covering more than $98 \%$ of the residents in Taiwan[22]. The NHI database was used to provide complete prescription records associated with all types of chemotherapy. The third database, the National Death Registry (NDR) database in Taiwan, was used to match for death events. All personal data were encrypted and analyzed anonymously. The release of all data used in this study was approved by the Data Release Review Board of the Collaboration Center of Health Information Application, Ministry of
Health and Welfare, Executive Yuan, Taiwan. The protocol for this study was approved by the Research Ethics Committee of National Taiwan University Hospital.

\section{Study Population and Variables}

We screened all of the patients registered in the TCRD between 2005 and 2009 for eligibility. The inclusion criteria included the following: 1. patients with histology-proven primary NSCLC (ICD-O-3 C339 and C34, excluding morphology codes of 8041, 8044 or 8045$)$; 2 . patients aged $\geq 18$ years old; 3 . patients who were initially diagnosed with advanced NSCLC, which was defined as stage IIIB or IV according to the American Joint Committee on Cancer (AJCC) $6^{\text {th }}$ edition[23]. Patients with missing clinical data or those who were diagnosed with hematologic malignancies (ICD-O-3 morphology code 9140, 9590-9989) were excluded.

Patients were deemed to have received chemotherapy if they were prescribed cytotoxic or targeted chemotherapy drugs, including pemetrexed, tegafur, gemcitabine, vinorelbine, etoposide, paclitaxel, docetaxel, epirubicin, cisplatin, carboplatin, bevacizumab, gefitinib, and erlotinib. All lines of chemotherapy were defined according to the inclusion of non-platinum drugs. Drugs prescribed continuously with no more than 2 months interruption were considered same-line therapy.

Patients enrolled in the study cohort were divided into three subgroups according to the histologic type: adenocarcinoma (ICD-O-3 morphology codes of 8140, 8141, 8143, 8250, 8255, $8260,8480,8481,8490$ ), SCC ( ICD-O-3 morphology codes of 8052, 8070, 8071, 8072, 8073, 8074, 8076, 8082, $8083)$, and other histologies. Utilization of first-line chemotherapy and overall survival (OS) were investigated in order to compare histologic subgroups. We also evaluated geographic differences in the use of chemotherapy by categorizing hospitals into five regions: Taipei, Northern, Central, Southern, and Kao-ping/Eastern (KPE) regions.

\section{Statistical Analysis}

Categorical variables were compared using the chi-square test. OS was calculated from the date of diagnosis for advanced NSCLC to the date of death or 31 December 2012, whichever came first. The data were regarded as censored if patients survived beyond the final date of the NDR database (December 31, 2012). OS was estimated using the Kaplan-Meier method, and the log-rank test was used to compare differences between groups. Two-sided $p$ values less than 0.05 were considered statistically significant. All analyses were performed using SAS software, Version 
9.3 (SAS Institute Inc., Cary, NC, USA).

\section{Results}

\section{Patient Demographics}

After identifying a total of 25,008 patients with advanced NSCLC, 17,443 (70.0\%) patients had received first-line chemotherapy and were therefore included in this study (Fig. 1). Among them, $60.5 \%$ of patients were male, $11,551(66.2 \%)$ patients had adenocarcinoma, and 3,292 (18.9\%) patients had SCC
(Table 1). Compared to patients with SCC, a greater number of those with adenocarcinoma were female (48\% vs. $14.8 \%, p<0.001)$, younger than 70 years $(63.7 \%$ vs. $51.4 \%, p<0.001)$, and initially diagnosed with stage IV disease $(77.8 \%$ vs. $58.9 \%, p<0.001)$. Total $68.4 \%$ of the patients were diagnosed with advanced NSCLC in medical centers. The Taipei region had the greatest number of patients (37.2\%) newly diagnosed with NSCLC.

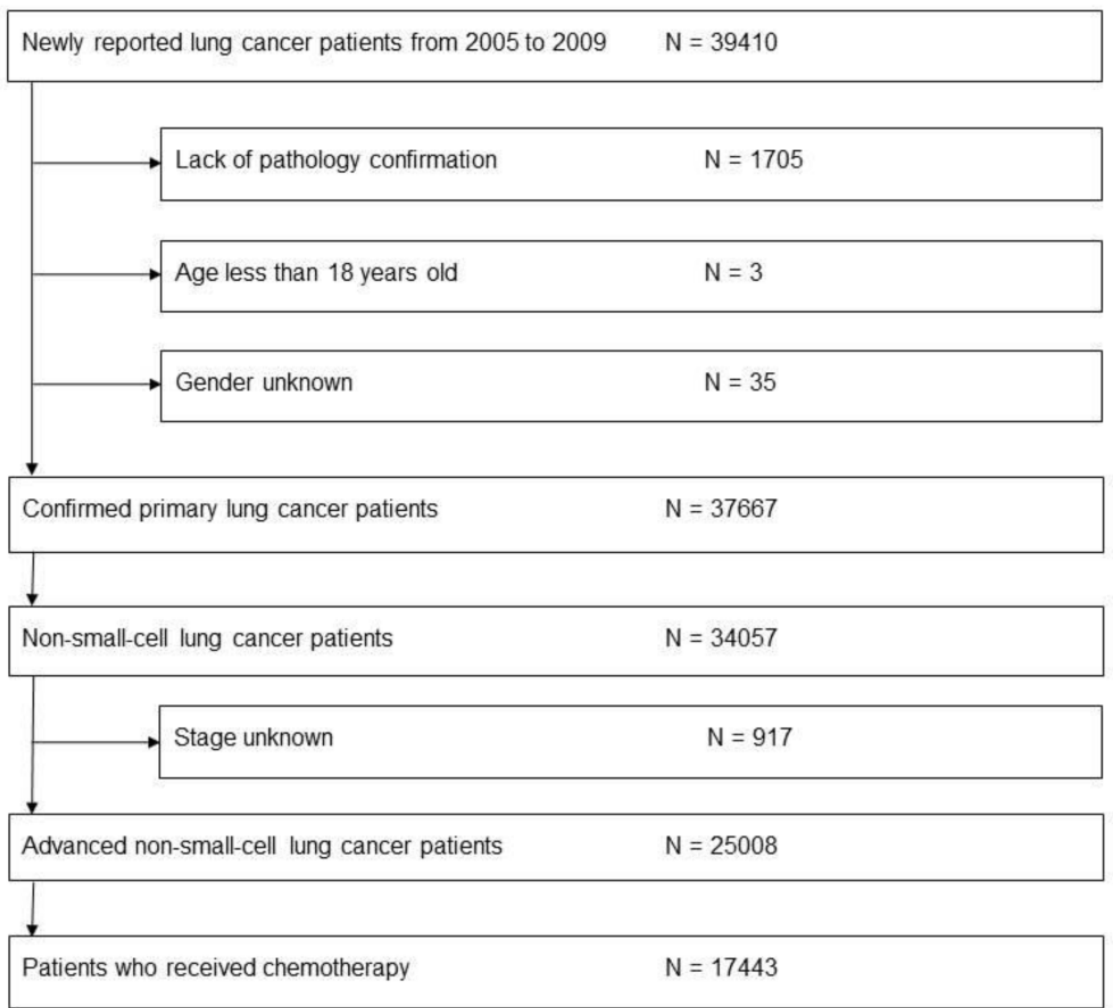

Fig 1: Flow chart illustrating process of evaluating patients for inclusion in study

Table 1: Clinical characteristics of patients

\begin{tabular}{|c|c|c|c|c|c|c|c|c|c|}
\hline \multirow[t]{2}{*}{ Characteristics } & & \multicolumn{2}{|l|}{ All } & \multicolumn{2}{|c|}{ Adenocarcinoma } & \multicolumn{2}{|l|}{ SCC } & \multicolumn{2}{|l|}{ Others } \\
\hline & & $\mathbf{N}$ & $\%$ & $\mathbf{N}$ & $\%$ & $\mathbf{N}$ & $\%$ & $\mathbf{N}$ & $\%$ \\
\hline Total & & 17,443 & $(100.0)$ & 11,551 & $(66.2)^{*}$ & 3,292 & $(18.9)^{*}$ & 2,600 & $(14.9)^{*}$ \\
\hline \multirow[t]{2}{*}{ Gender } & Male & 10,557 & $(60.5)$ & 5,975 & $(51.7)$ & 2,805 & $(85.2)$ & 1,777 & $(68.3)$ \\
\hline & Female & 6,886 & $(39.5)$ & 5,576 & $(48.3)$ & 487 & $(14.8)$ & 823 & $(31.7)$ \\
\hline Age & Mean (SD), years & $64.46(12.33)$ & & $63.54(12.53)$ & & 67.35 (11.08) & & $64.92(12.39)$ & \\
\hline \multirow[t]{2}{*}{ Age } & $<70$ & 10,569 & $(60.6)$ & 7,356 & $(63.7)$ & 1,691 & (51.4) & 1,522 & $(58.5)$ \\
\hline & $\geq 70$ & 6,874 & $(39.4)$ & 4,195 & $(36.3)$ & 1,601 & $(48.6)$ & 1,078 & $(41.5)$ \\
\hline \multirow[t]{2}{*}{ Stage } & IIIB & 4,650 & $(26.7)$ & 2,560 & $(22.2)$ & 1,352 & $(41.1)$ & 738 & $(28.4)$ \\
\hline & IV & 12,793 & (73.3) & 8,991 & $(77.8)$ & 1,940 & $(58.9)$ & 1,862 & $(71.6)$ \\
\hline \multirow[t]{5}{*}{ Year of diagnosis } & 2005 & 2,695 & $(15.5)$ & 1,671 & $(14.5)$ & 567 & $(17.2)$ & 457 & $(17.6)$ \\
\hline & 2006 & 2,953 & $(16.9)$ & 1,922 & $(16.6)$ & 621 & $(18.9)$ & 410 & $(15.8)$ \\
\hline & 2007 & 3,694 & $(21.2)$ & 2,425 & $(21.0)$ & 670 & $(20.4)$ & 599 & $(23.0)$ \\
\hline & 2008 & 3,873 & $(22.2)$ & 2,596 & $(22.5)$ & 716 & (21.7) & 561 & $(21.6)$ \\
\hline & 2009 & 4,228 & $(24.2)$ & 2,937 & $(25.4)$ & 718 & $(21.8)$ & 573 & $(22.0)$ \\
\hline \multirow[t]{2}{*}{ Facility Type } & Medical Center & 11,926 & $(68.4)$ & 8125 & $(70.3)$ & 2219 & $(67.4)$ & 1582 & $(60.8)$ \\
\hline & Non-Center & 5,517 & $(31.6)$ & 3,426 & $(29.7)$ & 1,073 & $(32.6)$ & 1,018 & $(39.2)$ \\
\hline
\end{tabular}

Abbreviations: SCC = squamous cell carcinoma;

*: This percentage was based on total 17,443 patients 


\section{First-Line Chemotherapy}

Platinum-based doublet chemotherapy was administered to $66.9 \%$ of patients (Table 2). Platinum with gemcitabine $(33.8 \%)$ was the most common chemotherapy regimen, followed by vinorelbine monotherapy $(13.0 \%)$ and platinum with docetaxel (11.6\%). Tumor histology had no obvious impact on the selection of chemotherapy regimens; however, patients with adenocarcinoma were more likely to receive subsequent chemotherapy than were patients with SCC (71.1\% vs. $54.6 \%, p<0.001)$.
Between 2005 and 2009, the prevalence of platinum-based doublet chemotherapy decreased from $71.4 \%$ to $64.1 \%$ despite an increase in the actual number of patients (Table 3). Platinum with gemcitabine presented the most pronounced decrease, from $37.8 \%$ in 2005 to $27.1 \%$ in 2009 ; however, it remained the most common regimen. Vinorelbine monotherapy increased rapidly from $4.9 \%$ in 2005 to $17.4 \%$ in 2009 . Within the study period, the number of patients receiving subsequent chemotherapy after first-line chemotherapy gradually increased from $61.6 \%$ in 2005 to $68.6 \%$ in 2009 .

Table 2: Regimens used in first-line chemotherapy

\begin{tabular}{|c|c|c|c|c|c|c|c|c|}
\hline \multirow[t]{2}{*}{ Regimen } & \multicolumn{2}{|c|}{ All } & \multicolumn{2}{|c|}{ Adenocarcinoma } & \multicolumn{2}{|c|}{ SCC } & \multicolumn{2}{|c|}{ Others } \\
\hline & $\mathbf{N}$ & $\%$ & $\mathbf{N}$ & $\%$ & $\mathbf{N}$ & $\%$ & $\mathbf{N}$ & $\%$ \\
\hline Total & 17,443 & $(100.0)$ & 11,551 & $(66.2) \#$ & 3,292 & $(18.9)^{\#}$ & 2,600 & $(14.9)^{\#}$ \\
\hline Platinum with & 11,678 & $(66.9)$ & 7,748 & $(67.1)$ & 2,210 & $(67.1)$ & 1,720 & $(66.2)$ \\
\hline gemcitabine & 5,893 & $(33.8)$ & 4,057 & $(35.1)$ & 1,045 & $(31.7)$ & 791 & $(30.4)$ \\
\hline docetaxel & 2,027 & $(11.6)$ & 1,263 & $(10.9)$ & 447 & $(13.6)$ & 317 & $(12.2)$ \\
\hline vinorelbine & 1,653 & $(9.5)$ & 1,049 & $(9.1)$ & 352 & $(10.7)$ & 252 & $(9.7)$ \\
\hline paclitaxel & 1,232 & $(7.1)$ & 794 & $(6.9)$ & 252 & $(7.7)$ & 186 & $(7.2)$ \\
\hline pemetrexed & 270 & (1.5) & 257 & $(2.2)$ & $\mathrm{NA}^{*}$ & $\mathrm{NA}^{*}$ & $\mathrm{NA}^{*}$ & $\mathrm{NA}^{*}$ \\
\hline etoposide & 141 & $(0.8)$ & 33 & $(0.3)$ & $\mathrm{NA}^{*}$ & $\mathrm{NA}^{*}$ & $\mathrm{NA}^{*}$ & $\mathrm{NA}^{*}$ \\
\hline others & 462 & (2.6) & 295 & $(2.6)$ & 83 & $(2.5)$ & 84 & $(3.2)$ \\
\hline Single agent & 5,144 & $(29.5)$ & 3,328 & $(28.8)$ & 1,016 & $(30.9)$ & 800 & $(30.8)$ \\
\hline vinorelbine & 2,275 & $(13.0)$ & 1,501 & $(13.0)$ & 446 & $(13.5)$ & 328 & $(12.6)$ \\
\hline gemcitabine & 1,692 & $(9.7)$ & 1,129 & $(9.8)$ & 299 & $(9.1)$ & 264 & $(10.2)$ \\
\hline docetaxel & 584 & $(3.3)$ & 334 & $(2.9)$ & 145 & $(4.4)$ & 105 & $(4.0)$ \\
\hline paclitaxel & 299 & $(1.7)$ & 192 & $(1.7)$ & 50 & $(1.5)$ & 57 & $(2.2)$ \\
\hline tegafur, combinations & 294 & $(1.7)$ & 172 & $(1.5)$ & 76 & $(2.3)$ & 46 & $(1.8)$ \\
\hline Others & 621 & $(3.6)$ & 475 & $(4.1)$ & 66 & $(2.0)$ & 80 & $(3.1)$ \\
\hline Subsequent Chemotherapy & 11,567 & (66.3) & 8,213 & (71.1) & 1,799 & $(54.6)$ & 1,555 & $(59.8)$ \\
\hline
\end{tabular}

Abbreviations: SCC = squamous cell carcinoma; NA: not accessible

*Due to personal privacy protection regulation, the numbers of these columns were not accessible

\#: This percentage was based on total 17,443 patients

Table 3: Regimens used in first-line chemotherapy according to yearly distribution

\begin{tabular}{|c|c|c|c|c|c|c|c|c|c|c|c|c|}
\hline \multirow[t]{2}{*}{ Regimen } & \multicolumn{12}{|l|}{$\mathbf{N}(\%)$} \\
\hline & Total & & 2005 & & 2006 & & 2007 & & 2008 & & 2009 & \\
\hline Total & 17,443 & $(100.0)$ & 2,695 & $(15.5)^{*}$ & 2,953 & $(16.9)^{*}$ & 3,694 & $(21.2)^{*}$ & 3,873 & $(22.2)^{*}$ & 4,228 & $(24.2)^{*}$ \\
\hline Platinum with & 11,678 & $(66.9)$ & 1,923 & $(71.4)$ & 1,999 & $(67.7)$ & 2,488 & $(67.4)$ & 2,557 & $(66.0)$ & 2,711 & $(64.1)$ \\
\hline gemcitabine & 5,893 & $(33.8)$ & 1,020 & $(37.8)$ & 1,076 & $(36.4)$ & 1,387 & $(37.5)$ & 1,265 & $(32.7)$ & 1,145 & $(27.1)$ \\
\hline docetaxel & 2,027 & $(11.6)$ & 288 & $(10.7)$ & 318 & $(10.8)$ & 395 & $(10.7)$ & 513 & $(13.2)$ & 513 & $(12.1)$ \\
\hline vinorelbine & 1,653 & $(9.5)$ & 295 & $(10.9)$ & 280 & (9.5) & 315 & $(8.5)$ & 368 & $(9.5)$ & 395 & $(9.3)$ \\
\hline paclitaxel & 1,232 & (7.1) & 185 & $(6.9)$ & 210 & $(7.1)$ & 287 & $(7.8)$ & 294 & (7.6) & 256 & (6.1) \\
\hline pemetrexed & 270 & (1.5) & & & & & & & & & 270 & $(6.4)$ \\
\hline etoposide & 141 & $(0.8)$ & 29 & (1.1) & 24 & $(0.8)$ & 23 & $(0.6)$ & 23 & $(0.6)$ & 42 & $(1.0)$ \\
\hline Others & 462 & $(2.6)$ & 106 & (3.9) & 91 & (3.1) & 81 & $(2.2)$ & 94 & $(2.4)$ & 90 & $(2.1)$ \\
\hline Single agent & 5,144 & (29.5) & 683 & $(25.3)$ & 849 & $(28.8)$ & 1,115 & $(30.2)$ & 1,193 & $(30.8)$ & 1,304 & $(30.8)$ \\
\hline vinorelbine & 2,275 & $(13.0)$ & 132 & $(4.9)$ & 275 & $(9.3)$ & 506 & $(13.7)$ & 626 & $(16.2)$ & 736 & $(17.4)$ \\
\hline gemcitabine & 1,692 & $(9.7)$ & 294 & $(10.9)$ & 301 & $(10.2)$ & 367 & $(9.9)$ & 363 & $(9.4)$ & 367 & $(8.7)$ \\
\hline docetaxel & 584 & (3.3) & 130 & $(4.8)$ & 99 & (3.4) & 117 & $(3.2)$ & 116 & $(3.0)$ & 122 & $(2.9)$ \\
\hline paclitaxel & 299 & $(1.7)$ & 40 & $(1.5)$ & 104 & (3.5) & 77 & $(2.1)$ & 47 & $(1.2)$ & 31 & $(0.7)$ \\
\hline tegafur, combinations & 294 & $(1.7)$ & 87 & $(3.2)$ & 70 & $(2.4)$ & 48 & (1.3) & 41 & $(1.1)$ & 48 & $(1.1)$ \\
\hline Others & 621 & (3.6) & 89 & $(3.3)$ & 105 & $(3.6)$ & 91 & $(2.5)$ & 123 & $(3.2)$ & 213 & $(5.0)$ \\
\hline Subsequent Chemotherapy & 11,567 & $(66.3)$ & 1,659 & $(61.6)$ & 1,846 & $(62.5)$ & 2,473 & $(66.9)$ & 2,687 & $(69.4)$ & 2,902 & $(68.6)$ \\
\hline
\end{tabular}

*: This percentage was based on total 17,443 patients 
Table 4: Regimens used in first-line chemotherapy according to geographic distribution

\begin{tabular}{|c|c|c|c|c|c|c|c|c|c|c|c|c|}
\hline & N (\%) & & & & & & & & & & & \\
\hline Regimen & All & & Taipei & & Northern & & Central & & Southern & & KPE & \\
\hline Total & 17,443 & (100.0) & 6,494 & $(37.2)^{*}$ & 1,910 & $(10.9)^{*}$ & 3,434 & $(19.7)^{*}$ & 2,888 & $(16.6)^{*}$ & 2,717 & $(15.6)^{*}$ \\
\hline Platinum with & 11,678 & $(66.9)$ & 4,147 & $(63.9)$ & 1,372 & $(71.8)$ & 2,234 & $(65.1)$ & 2,008 & $(69.5)$ & 1,917 & $(70.6)$ \\
\hline gemcitabine & 5,893 & $(33.8)$ & 1,936 & $(29.8)$ & 503 & $(26.3)$ & 1,485 & $(43.2)$ & 1067 & $(36.9)$ & 902 & $(33.2)$ \\
\hline docetaxel & 2,027 & (11.6) & 690 & (10.6) & 408 & $(21.4)$ & 262 & (7.6) & 402 & (13.9) & 265 & (9.8) \\
\hline vinorelbine & 1,653 & (9.5) & 552 & (8.5) & 232 & $(12.1)$ & 136 & $(4.0)$ & 287 & (9.9) & 446 & (16.4) \\
\hline paclitaxel & 1,232 & (7.1) & 567 & (8.7) & 152 & $(8.0)$ & 207 & $(6.0)$ & 112 & (3.9) & 194 & (7.1) \\
\hline pemetrexed & 270 & (1.5) & 137 & (2.1) & 19 & $(1.0)$ & 72 & $(2.1)$ & 24 & $(0.8)$ & 18 & $(0.7)$ \\
\hline etoposide & 141 & $(0.8)$ & 59 & $(0.9)$ & 12 & $(0.6)$ & 14 & $(0.4)$ & 26 & $(0.9)$ & 30 & (1.1) \\
\hline others & 462 & (2.6) & 206 & (3.2) & 46 & (2.4) & 58 & (1.7) & 90 & (3.1) & 62 & (2.3) \\
\hline Single agent & 5,144 & (29.5) & 2,013 & $(31.0)$ & 506 & $(26.5)$ & 1,072 & $(31.2)$ & 813 & $(28.2)$ & 740 & $(27.2)$ \\
\hline vinorelbine & 2,275 & $(13.0)$ & 1,021 & $(15.7)$ & 228 & $(11.9)$ & 295 & $(8.6)$ & 434 & $(15.0)$ & 297 & $(10.9)$ \\
\hline gemcitabine & 1,692 & $(9.7)$ & 557 & $(8.6)$ & 139 & $(7.3)$ & 503 & $(14.6)$ & 213 & (7.4) & 280 & $(10.3)$ \\
\hline docetaxel & 584 & (3.3) & 216 & (3.3) & 56 & (2.9) & 157 & $(4.6)$ & 56 & (1.9) & 99 & (3.6) \\
\hline paclitaxel & 299 & (1.7) & 115 & (1.8) & 65 & (3.4) & 48 & (1.4) & 34 & (1.2) & 37 & (1.4) \\
\hline tegafur, combinations & 294 & (1.7) & 104 & (1.6) & 18 & $(0.9)$ & 69 & $(2.0)$ & 76 & $(2.6)$ & 27 & $(1.0)$ \\
\hline Others & 621 & (3.6) & 334 & (5.1) & 32 & (1.7) & 128 & (3.7) & 67 & (2.3) & 60 & $(2.2)$ \\
\hline
\end{tabular}

Abbreviations: KPE = Kaohsiung, Ping-Tung, and Eastern

*: This percentage was based on total 17,443 patients

Irrespective of geographic region, platinum with gemcitabine was the most common first-line chemotherapy regimen administered in Taiwan (Table 4). The prevalence of this regimen was highest in central Taiwan $(43.2 \%)$ and southern Taiwan $(36.9 \%)$. Platinum with docetaxel was more commonly used in the Northern region (21.4\%) compared to other regions. Platinum with vinorelbine was more commonly used in the KPE region (16.4\%). Physicians in the Taipei and Central regions were more likely to opt for single agents as first-line treatment, compared to physicians in other regions. Among the single agents, physicians in the Taipei, Northern, and Southern regions preferred vinorelbine, whereas physicians in central Taiwan preferred gemcitabine.

\section{Survival}

As of December 31, 2012, with a median follow-up of 14 months, 15,953 (91\%) patients had died. The median OS (mOS) of all patients with advanced NSCLC was 13.9 months (95\% confidence interval [CI]: 13.6 -14.2 months). Patients with adenocarcinoma had significantly longer mOS than did patients with SCC or NSCLC with other histologies (mOS: 16.4 vs. 10.2 vs. 10.1 months, $p<$ 0.001, Fig. 2).

Among patients who received first-line platinum-based doublet chemotherapy for advanced lung adenocarcinoma, those who were administered platinum with pemetrexed exhibited significantly longer mOS (27.1 months) than did patients who received other regimens (mOS: 16.6-19.3 months, $p<$ 0.001; Fig. 3A). Patients who were administered platinum with gemcitabine presented similar mOS (18.6 months, 95\% CI: 18.0-19.3 months) comparing to those who received platinum with docetaxel (mOS: 17.4 months, 95\% CI: 16.3-18.7 months) and those who received platinum with vinorelbine (mOS: 16.6 months, 95\% CI: 15.5-17.6 months). However, platinum with pemetrexed was not approved as first-line therapy by the NHI until 2009. Even among patients diagnosed with advanced NSCLC in 2009, the mOS of patients who received platinum with pemetrexed was still significant longer than that of patients who received platinum with vinorelbine (mOS: 18.6 months, $p<0.001$; Fig. 3B), platinum with gemcitabine (mOS: 18.4 months, $p<0.001$ ), or platinum with docetaxel (mOS: 15.9 months, $p<$ 0.001). Patients who received platinum with paclitaxel and those who received platinum with pemetrexed had similar mOS $(p=0.340)$. Among patients who received single-agent regimens as first-line chemotherapy for advanced lung adenocarcinoma, those who received vinorelbine (mOS:12.5 months, 95\% CI: 11.7-13.6 months) exhibited significantly longer mOS than did patients who received gemcitabine (10.6 months, 95\% CI: 9.7-11.6 months, $p$ $=0.003)$ or tegafur-containing medications (10.3 months, 95\% CI: 8.7-12.2 months, $p=0.014$ ); however, their mOS was similar to that of patients who received docetaxel (11.0 months, 95\% CI: 9.2-13.1 months, $p$ $=0.750)$ or paclitaxel (11.6 months, 95\% CI: 9.3-15.7 months, $p=0.601$, Fig. 3C).

Among patients who received first-line platinum-based doublet chemotherapy for advanced lung SCC, those who received platinum with docetaxel presented significantly longer mOS (13.2 months, 95\% CI: 10.9-15.1 months) than did patients who received platinum with gemcitabine (11.4 months, 95\% CI: 10.6-12.0 months, $p<0.001)$ or vinorelbine (11.5 months, 95\% CI: 9.6-12.5 months, $p=$ 
0.001; Fig. 3D); however, their mOS was similar to that of patients who received platinum with paclitaxel (11.8 months, 95\% CI: 10.5-13.3 months, $p=0.326$ ). Among patients who received single-agent regimens as first-line chemotherapy for advanced lung SCC, gemcitabine resulted in mOS values significantly shorter than those achieved by using other single agents $(p<0.001$, Fig 3E). Except for gemcitabine, the other four agents all demonstrated similar mOS to each other $(p=0.709)$.

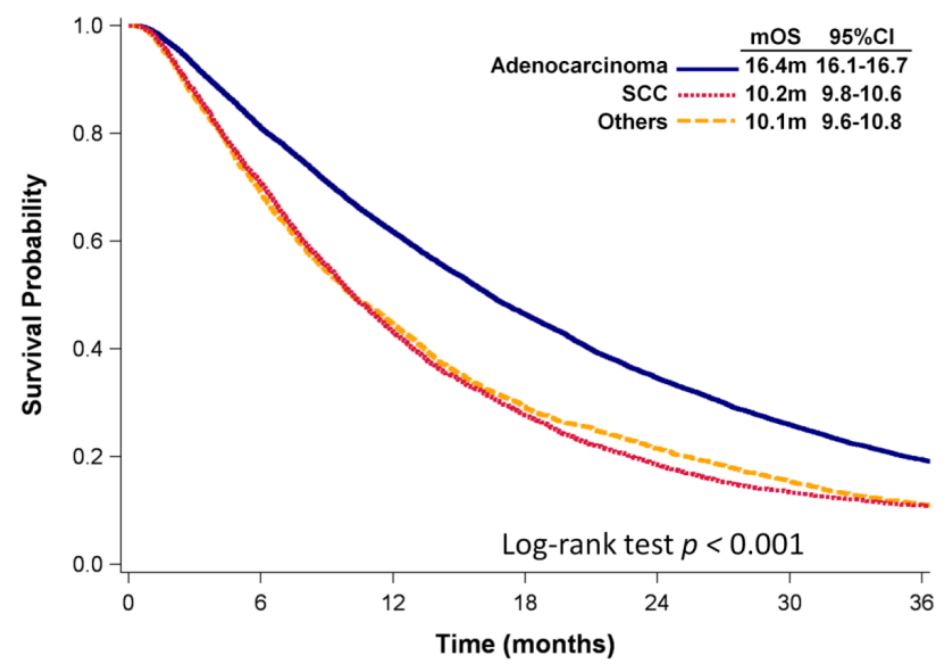

Fig 2: Kaplan-Meier plots of OS of patients with various NSCLC histologies
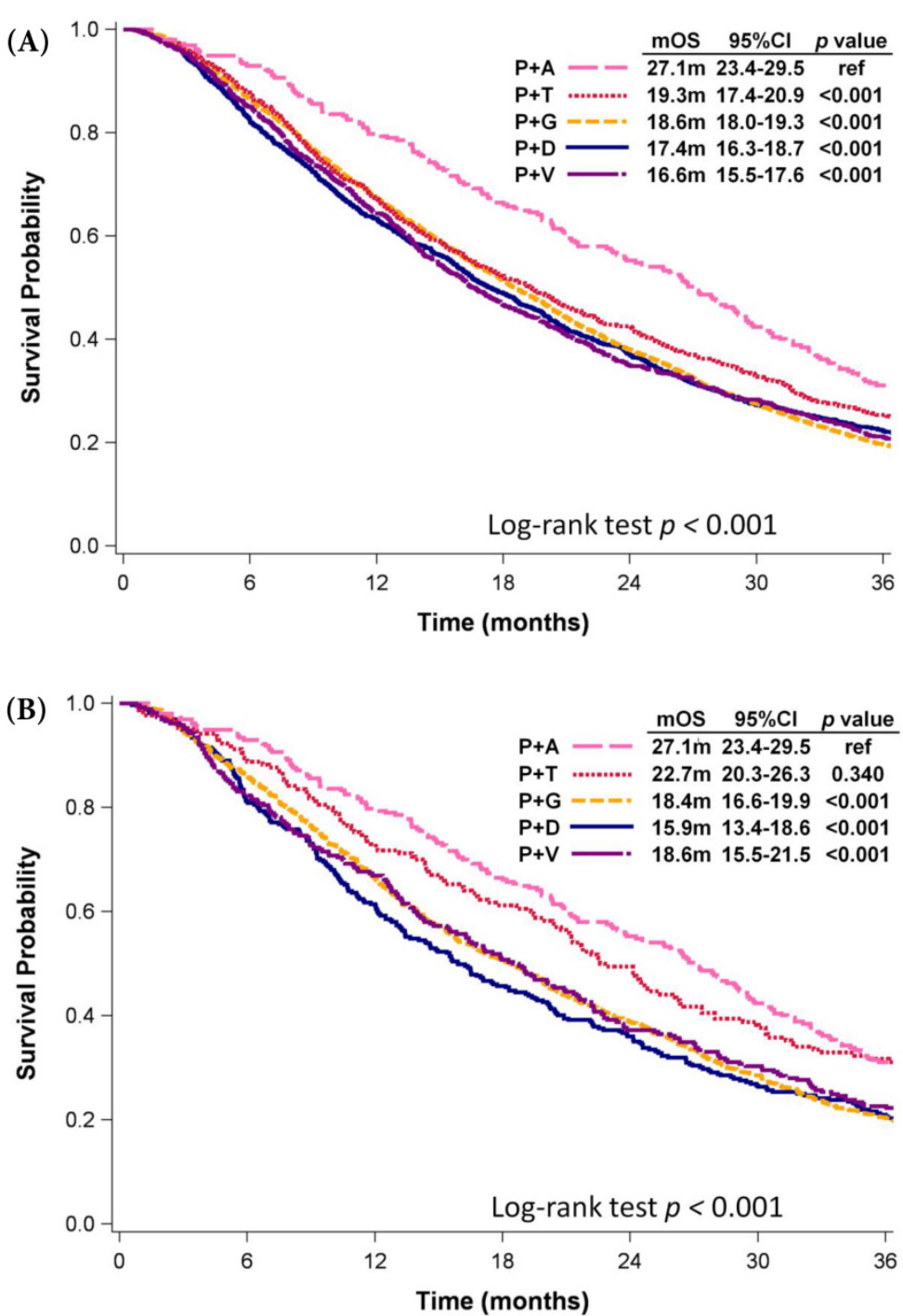

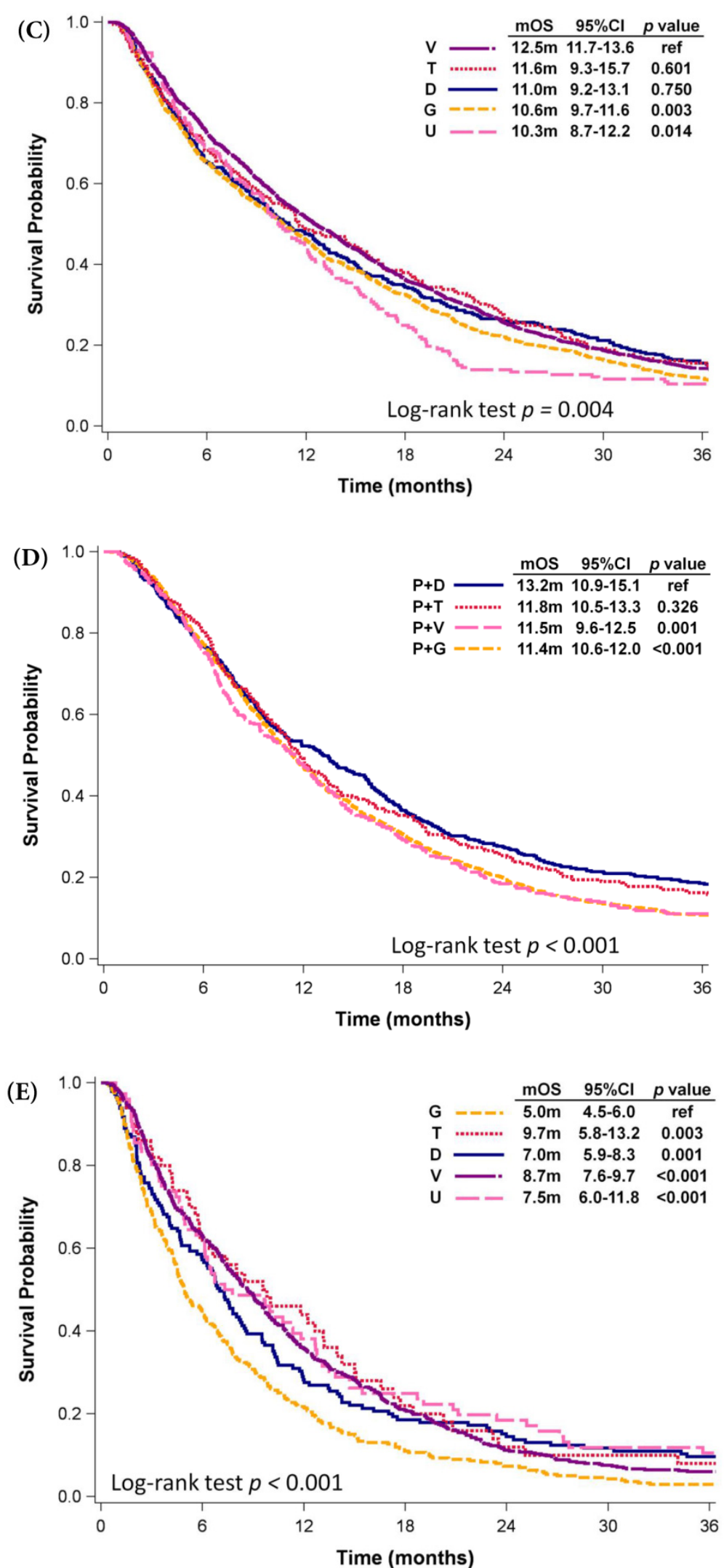

Fig 3: Kaplan-Meier plots of overall survival of (A) patients with adenocarcinoma who were administered various first-line platinum-based doublet chemotherapy regimens; (B) patients with adenocarcinoma who received various first-line platinum-based doublet chemotherapy regimens in 2009; (C) patients with adenocarcinoma who received various single agents as first-line therapy; (D) patients with SCC who received various first-line platinum-based doublet chemotherapy regimens; (E) patients with SCC who received various single agents as first-line therapy. Abbreviations: P: Platinum; A: Pemetrexed; D: Docetaxel; G: Gemcitabine; T: Paclitaxel; V: Vinorelbine; U: Tegafur/uracil; mOS: median overall survival; $95 \% \mathrm{Cl}: 95 \%$ confidence interval; ref: reference value 


\section{Discussion}

In this study, approximately $70 \%$ of patients with advanced NSCLC in Taiwan received first-line chemotherapy. Among them, 66.9\% received platinum-based doublet chemotherapy. Patients with adenocarcinoma had significantly longer mOS than did patients with other histologies. Platinum with gemcitabine was the most common first-line regimen in Taiwan, irrespective of geographic region. EGFR inhibitors as first-line therapy were not yet being reimbursed by the NHI during the study period; therefore, we did not analyze these compounds.

Platinum with gemcitabine was shown to be the most common first-line chemotherapy regimen in Taiwan; however, various other regimens were more common in specific regions, such as platinum with docetaxel in northern Taiwan and platinum with vinorelbine in the KPE region. Here existed several explanations. First, histology distribution differed among the various geographic regions; i.e., $11.1 \%$ of the patients with advanced NSCLC in the central region were diagnosed with other histologies, compared to $20.5 \%$ of the patients in the southern region. By contrast, $70.2 \%$ of the patients in the Taipei region were diagnosed with adenocarcinoma, compared to around $60 \%$ of the patients in the northern and southern regions. Second, physicians and hospitals may have been following established standards with regard to first-line regimens, based on the fact that many clinical trials have proved that most types of first-line chemotherapy provide similar survival benefits[13, 14, 17, 19, 24].

Among patients with advanced lung adenocarcinoma, those who were administered platinum with pemetrexed exhibited longer mOS than did patients who received other first-line platinum-based doublets chemotherapy. This is comparable to the results of previous clinical trials and retrospective studies[8, 25-27]. Pemetrexed was not approved as first-line chemotherapy for advanced lung adenocarcinoma until 2009; therefore, we restricted our analysis to patients who were diagnosed in 2009. Nonetheless, the results were much the same. An mOS of 27.1 months among patients who received platinum with pemetrexed was surprisingly good, compared to historical controls $[8$, 25-28]. This may be related to the high prevalence of EGFR mutations among cases of adenocarcinoma in Taiwan as well as the reimbursement of EGFR inhibitors as salvage therapy by the NHI. Overall, $63.4 \%$ of patients who received platinum with pemetrexed as first-line chemotherapy were subsequently reimbursed with EGFR inhibitors.
Nearly $30 \%$ of the patients received single agent chemotherapy as first-line therapy in Taiwan. For patients who were considered unfit for platinum-based chemotherapy due to poor performance or advanced age, this approach is reasonable. Meta-analysis revealed that for elderly patients with late stage NSCLC, doublet chemotherapy did not extend OS beyond that achieved by using single-agent chemotherapy[29]. Among the various single agents, a clinical trial in which only patients with poor performance status were enrolled presented no significant difference between vinorelbine and paclitaxel. Our results show that patients with adenocarcinoma who received vinorelbine alone had better OS than did patients who received gemcitabine or tegafur/uracil. However, selection bias may have a strong influence on this finding. Whether vinorelbine is indeed a better choice for patients considering single-agent therapy remains to be clarified.

The gradual decrease in the use of first-line platinum-based doublet chemotherapy within the study period may be associated with the emergence of targeted chemotherapy, such as EGFR inhibitors. Although gefitinib as salvage therapy for advanced NSCLC was reimbursed by the NHI, it was not approved as first-line therapy until 2011. This study was unable to monitor prescription patterns that did not fall within the scope of NHI reimbursement. The use of EGFR-targeted therapy may have resulted in a decline in the use of platinum-based chemotherapy.

This study has a number of limitations. As mentioned previously, we were unable to analyze prescriptions that are not covered by the NHI, such as when medication is paid for by the patients themselves or in the case of clinical trials. We were also unable to access data related to the performance status of patients, which probably exerted a significant impact on the selection of chemotherapy regimens. History of tobacco use was not recorded in the databases as well. The impacts of certain important comorbidity diseases, such as chronic obstructive pulmonary disease and diabetes, were not clear. Furthermore, EGFR mutation and other gene alterations status were not available in our data.

In conclusion, we found that platinum with gemcitabine was the most common first-line chemotherapy regimen for patients with advanced NSCLC in Taiwan, irrespective of histologic type and geographic region. Among patients with adenocarcinoma histology, those who received platinum with pemetrexed presented longer OS than did patients who received other platinum-based regimens. 


\section{Acknowledgement}

The study was supported by the Science and Technology Unit, Ministry of Health and Welfare, Taiwan (Grant No. DOH102-NH-9002).

\section{Competing Interests}

The authors have declared that no competing interest exists.

\section{References}

1. WHO. Mortality burden of disease -- Cause specific mortality, 2008. World Health Organization. 2012.

2. Hsiao AJ, Chen $\mathrm{LH}, \mathrm{Lu}$ TH. Ten leading causes of death in Taiwan: A comparison of two grouping lists. J Formos Med Assoc. 2014.

3. Chien CR, Tsai CM, Tang ST, Chung KP, Chiu CH, Lai MS. Quality of care for lung cancer in Taiwan: a pattern of care based on core measures in the Taiwan Cancer Database registry. J Formos Med Assoc. 2008; 107: 635-43.

4. Yang JC, Kang JH, Mok T, Ahn MJ, Srimuninnimit V, Lin CC, et al. First-line pemetrexed plus cisplatin followed by gefitinib maintenance therapy versus gefitinib monotherapy in East Asian patients with locally advanced or metastatic non-squamous non-small cell lung cancer: a randomised, phase 3 trial. Eur J Cancer. 2014; 50: 2219-30.

5. Yang JC, Shih JY, Su WC, Hsia TC, Tsai CM, Ou SH, et al. Afatinib for patients with lung adenocarcinoma and epidermal growth factor receptor mutations (LUX-Lung 2): a phase 2 trial. Lancet Oncol. 2012; 13: 539-48.

6. Mitsudomi T, Morita S, Yatabe Y, Negoro S, Okamoto I, Tsurutani J, et al. Gefitinib versus cisplatin plus docetaxel in patients with non-small-cell lung cancer harbouring mutations of the epidermal growth factor receptor (WJTOG3405): an open label, randomised phase 3 trial. Lancet Oncol. 2010; 11: $121-8$.

7. Mok TS, Wu YL, Thongprasert S, Yang CH, Chu DT, Saijo N, et al. Gefitinib or carboplatin-paclitaxel in pulmonary adenocarcinoma. N Engl J Med. 2009; 361: 947-57.

8. Scagliotti GV, Parikh P, von Pawel J, Biesma B, Vansteenkiste J, Manegold C, et al. Phase III study comparing cisplatin plus gemcitabine with cisplatin plus pemetrexed in chemotherapy-naive patients with advanced-stage non-small-cell lung cancer. J Clin Oncol. 2008; 26: 3543-51.

9. Ohe Y, Ohashi Y, Kubota K, Tamura T, Nakagawa K, Negoro S, et al. Randomized phase III study of cisplatin plus irinotecan versus carboplatin plus paclitaxel, cisplatin plus gemcitabine, and cisplatin plus vinorelbine for advanced non-small-cell lung cancer: Four-Arm Cooperative Study in Japan. Ann Oncol. 2007; 18: 317-23.

10. D'Addario G, Pintilie M, Leighl NB, Feld R, Cerny T, Shepherd FA. Platinum-based versus non-platinum-based chemotherapy in advanced non-small-cell lung cancer: a meta-analysis of the published literature. J Clin Oncol. 2005; 23: 2926-36.

11. Belani CP, Fossella F. Elderly subgroup analysis of a randomized phase III study of docetaxel plus platinum combinations versus vinorelbine plus cisplatin for first-line treatment of advanced nonsmall cell lung carcinoma (TAX 326). Cancer. 2005; 104: 2766-74.

12. Hanna N, Shepherd FA, Fossella FV, Pereira JR, De Marinis F, von Pawel J, et al. Randomized phase III trial of pemetrexed versus docetaxel in patients with non-small-cell lung cancer previously treated with chemotherapy. J Clin Oncol. 2004; 22: 1589-97.

13. Fossella F, Pereira JR, von Pawel J, Pluzanska A, Gorbounova V, Kaukel E, et al. Randomized, multinational, phase III study of docetaxel plus platinum combinations versus vinorelbine plus cisplatin for advanced non-small-cell lung cancer: the TAX 326 study group. J Clin Oncol. 2003; 21: 3016-24.

14. Schiller JH, Harrington D, Belani CP, Langer C, Sandler A, Krook J, et al. Comparison of four chemotherapy regimens for advanced non-small-cell lung cancer. N Engl J Med. 2002; 346: 92-8.

15. Kelly K, Crowley J, Bunn PA, Jr., Presant CA, Grevstad PK, Moinpour CM, et al. Randomized phase III trial of paclitaxel plus carboplatin versus vinorelbine plus cisplatin in the treatment of patients with advanced non--small-cell lung cancer: a Southwest Oncology Group trial. J Clin Oncol. 2001; 19: 3210-8.

16. Sandler AB, Nemunaitis J, Denham C, von Pawel J, Cormier Y, Gatzemeier U, et al. Phase III trial of gemcitabine plus cisplatin versus cisplatin alone in patients with locally advanced or metastatic non-small-cell lung cancer. J Clin Oncol. 2000; 18: 122-30.

17. Scagliotti GV, De Marinis F, Rinaldi M, Crino L, Gridelli C, Ricci S, et al. Phase III randomized trial comparing three platinum-based doublets in advanced non-small-cell lung cancer. J Clin Oncol. 2002; 20: 4285-91.

18. Chemotherapy in non-small cell lung cancer: a meta-analysis using updated data on individual patients from 52 randomised clinical trials. Non-small Cell Lung Cancer Collaborative Group. BMJ. 1995; 311: 899-909.

19. Liao BC, Shao YY, Chen HM, Shau WY, Lin ZZ, Kuo NC, Lai CL, Chen KH, Cheng AL, Yang CH. Comparative Effectiveness of First-Line Platinum-Based Chemotherapy Regimens for Advanced Lung Squamous Cell Carcinoma. Clinical Lung Cancer. 2014.
20. [Internet] Taiwan Cancer Registry. http://tcr.cph.ntu.edu.tw/ main.php?Page $=\mathrm{N} 1$

21. Chiang CJ, Lo WC, Yang YW, You SL, Chen CJ, Lai MS. Incidence and Survival of Adult Cancer Patients in Taiwan, 2002-2012. J Formos Med Assoc. 2016; Jan 16; pii:S0929-6646(15)00349-6.

22. [Internet] Taiwan's National Health Insurance (NHI) Administration. http://www.nhi.gov.tw/english/index.aspx. 2016.

23. Greene F, Page D, Fleming I, et al. American Joint Committee on Cancer (AJCC). Cancer Staging Handbook 6th ed New York: Springer. 2002.

24. Clements KM, Peltz G, Faries DE, Lang K, Nyambose J, Earle CC, et al. Does Type of Tumor Histology Impact Survival among Patients with Stage IIIB/IV Non-Small Cell Lung Cancer Treated with First-Line Doublet Chemotherapy? Chemother Res Pract. 2010; 2010: 524629.

25. Treat I, Scagliotti GV, Peng G, Monberg MJ, Obasaju CK, Socinski MA. Comparison of pemetrexed plus cisplatin with other first-line doublets in advanced non-small cell lung cancer (NSCLC): a combined analysis of three phase 3 trials. Lung Cancer. 2012; 76: 222-7.

26. Wu YL, Lu S, Cheng Y, Zhou C, Wang M, Qin S, et al. Efficacy and safety of pemetrexed/cisplatin versus gemcitabine/cisplatin as first-line treatment in Chinese patients with advanced nonsquamous non-small cell lung cancer. Lung Cancer. 2014; 85: 401-7.

27. Lee JH, Yu CJ, Chen KY, Shih JY, Lin YL, Yang CH. Pemetrexed for heavily pretreated patients with advanced non-small cell lung cancer. J Formos Med Assoc. 2010; 109: 338-44.

28. Scagliotti G, Hanna N, Fossella F, Sugarman K, Blatter J, Peterson P, et al. The differential efficacy of pemetrexed according to NSCLC histology: a review of two Phase III studies. Oncologist. 2009; 14: 253-63.

29. Des Guetz G, Uzzan B, Nicolas P, Valeyre D, Sebbane G, Morere JF. Comparison of the efficacy and safety of single-agent and doublet chemotherapy in advanced non-small cell lung cancer in the elderly: a meta-analysis. Crit Rev Oncol Hematol. 2012; 84: 340-9. 